\title{
رؤية مستقبلية لتطوير تعليم الموهوبين في مصر في ضوء
}

\section{الخبرة اليابانية}

\author{
إعداد \\ أ.د / محمد النصر حسن \\ رئيس قسم اصول التربية \\ كلية التربية بقنا- جامعة جنوب الوادي \\ melnasr@yahoo.com

\section{أ/حناز بوسف محمد سعدالأه} \\ باحثة ماجستير - قسم أصول التربية \\ كلية التربية بقنا- جامعة جنوب الوادي \\ hanan.you33@gmail.com
}

أ.د فثتحي عبد الرسول محمد

أستاذ أصول التربية المتفرغ

كلية التربية بقنا - جامعة جنوب الوادي

Fathe55555@yahoo.com 


\section{رؤية مستقبلية لتطوير تعليم الموهوبين في مصر في ضوء الخبرة اليابانية}

أ. د/ محمد النصر حسن

رئيس قسم اصول التربية

كلية التربية بقنا- جامعة جنوب الوادي

melnasr@yahoo.com
أ.د/فتحي عبد الرسول محمد

أستاذ أصول التربية المتفرغ

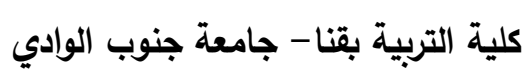

Fathe55555@yahoo.com

أ/حنان يوسف محمد سعدالله

باحثة ماجستير - قسم أصول التربية

hanan.you33@gmail.com

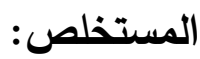

هدفت الدراسة إلى اقتراح رؤية مستقبلية لاكتشاف ورعاية الموهوبين في مصر في ضوء التجربة اليابانية، وذلك بعد التعرف إلى نظام اكتشاف الطلبة الموهوبين في اليابان ورعايتهم، وقد استخدت الباحثة المنهج الوصفي ، وتوصلت الدراسة إلى مجموعة من النتائج أهها: قصور واضح في نظام اكتشاف ورعاية الطلبة الموهوبين في مصر ، ويعتمد اختيار الموهوبين واكتشافهم على الدرجات التحصيلية، وتتشابه كل من مصر واليابان في الاهتمام بالموهوبين، ولكن تختلف في عدم تطبيق السياسات لاعتبارات متعلقة بالامكانات. وفي ضوء هذه النتائج تم اقتراح رؤية مستقبلية للاستفادة من الخبرة اليابانية في هذا المجال، وقد أوصت الباحثة بالآتي: الاهتمام بالاكتشاف المبكر للموهوبين. وأن يتم استخدام أساليب، وأدوات مقننه في عملية اكتشاف الطلبة الموهوبين في المراحل التعليمية المختلفة، عقد مسابقات تتظمها وزارة التربية والتعليم بالاشتراك مع وزارات أخرى بغرض اكتشاف المواهب وتتميتها ورعايتها. أن تقوم الجامعات المصرية بإعداد برامج خاصة باكتشاف الموهوبين. الكلمات المفتاحية: رؤية مستقبلية - تطوير - تعليم الموهوبين 
مما لاشك فيه أن العالم اليوم يشهد تقدمًا علميًا متسارعًا، لمواجهة تحديات القرن الواحد والعشرين، والذي يمتاز بالتحولات المعرفية، والاقتصادية، والتكنولوجية، ولمواكبة هذا

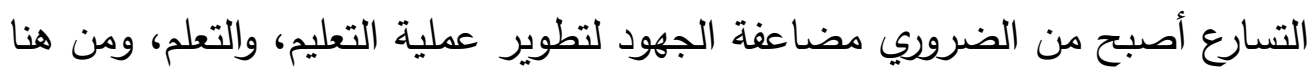
تقع المسؤولية على العاملين في التربية والتعليم للقيام بهذا التطور، ونتيجة لهذه الثورة العلمية، والتكنولوجية أصبح تطوير التعليم وجودته ضرورة ملحة كعامل أساسي في في التقدم، والتتمية الثاملة. حيث يعد التعليم اليوم من أبرز الموضوعات التي تشغل بال وجله السياسيين، والتربويين والمفكرين وغيرهم من المهتمين بقضايا التطوير والتتمية لما يقوم به من دور فعال في اكتشاف ورعاية وتدعيم وتعظيم القوى البشرية، والخبرات، والقدرات التي يمتلكها الإنسان، وتوجيهها بما يخدم الإنسان نفسه والمجتمع الذي يعيش

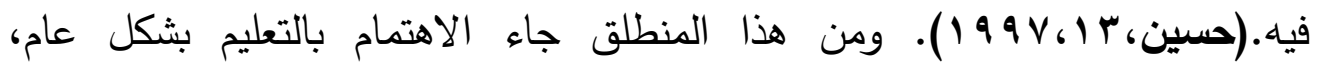
والاهتمام بتعليم الموهوبين بشكل خاص.

إن الموهوبون من أهم الفئات التي اهتمت بها التربية، والقائمين عليها، لتنمية مهاراتهم.حيث أن الاهتمام بالموهوبين قديم قدم الإنسان على مر العصور، كاهتمام الرومان بإعداد القادة العسكرين وعناية اليونان بالفلاسفة ورعاية الصين للحكماء،

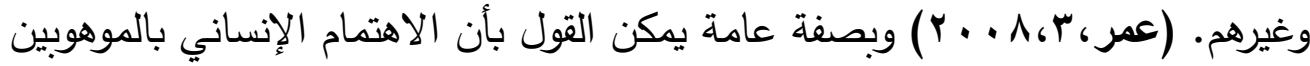

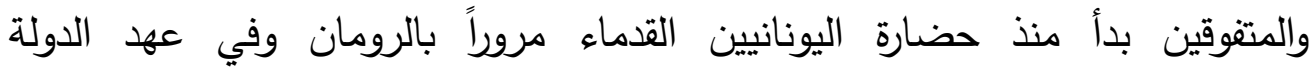
الإسلامية، إلى العصور الوسطى والحديثة في أوروبا وأمريكا وفي العصر الحديث بدأ الاهتمام بالمتفوقين والموهوبين في الدول العربية عام 1971 عندما أقر مؤتمر وزراء التربية العرب المنعقد في الكويت إقامة ندوة تربية الموهوبين والمتفوقين في الدول العربية، والتي أوصت بإنشاء فصول أو مدارس خاصة بتربية الموهوبين ووضع مناهج

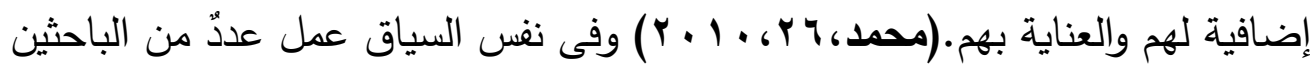
والمربين في قضية التربية الخاصة للموهوبين والمتفوقين بكل الوسائل المككنة، وكان لهم أثر كبير في تسليط الأضواء على هذه الثريحة، وربما كان من غير الممكن 
حصر جميع الإسهامات التي قدموها لهذه القضية في مختلف أنحاء العالم ومن أمثلتهم" لويس تيرمان، جالتون، وبينيه، وجوليان، ستانلي".(عمر ، مرجع سابق، Y r) لذا توجد محاولات جادة وصادقة نحو تقديم ما هو أفضل تمثل في التجارب الرائدة لبعض الدول تعتبر نماذج يمكن الاستفادة منها في تطوير النظم التربوية والخدمات الخاصة التي يمكن أن تسهم في رعاية الموهوبين في دول أو مجتمعات أخرى.

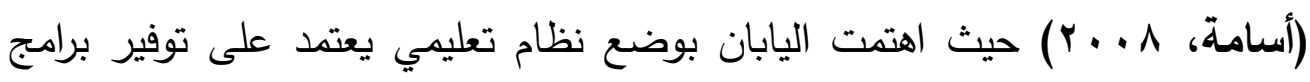

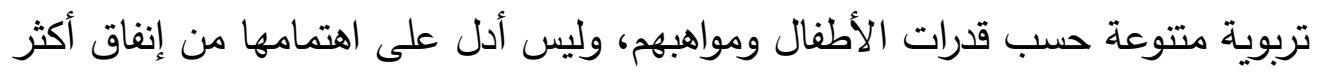

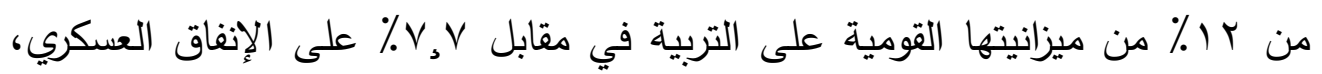
وقد انعكس ذلك على تصدر اليابان في مجالات علمية وتكنولوجية وتربوية. (زكريا

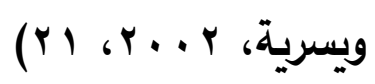

إن وجود استراتيجية لرعاية الموهوبين في مصر أصبحت ضرورة حتمية، للتخلص من العشوائيه التي ينتج عنها إهدار لطاقات وقدرات هولاء الثباب، ويصبح وجودهم في نظام تعليمي لا يلبي احتياجاتهم، ولا يوفر لهم الرعاية المناسبة للاستفادة من تلك الطاقات، ويتطلب ذلك تكاتف جميع مؤسسات الدولة الحكومية، والخاصة بتوفير نظام تعليمي يخدم هؤلاء الفئة، ويصبح العائد هو تطور المجتمع، ووضع الدوله في مقدمة الدول في جميع المجالات. لذا جاءت هذه الدراسة لوضع رؤية مستقبلية مقترحة لتعليم الطلاب الموهوبين في ضوء الخبرات العالمية الرائدة في هذا المجال. مشكلة الدراسة:

إن الموهوبين في المدارس المصرية، لم يحصلوا على القدر الكافي من العناية والتوجياه، كما أن تعليم الموهوبين في مصر يفتقر إلى طرق الكثف المبكرة للحفاظ على الموهوبين وتتمية قدراتهم، وتوفير الاككانات اللازمة للحفاظ على مواهبهم وتتميتها ومتابعتها في المراحل الدراسية المختلفة. وتتحدد مشكلة الدراسة الحالية في السؤال الرئيس التالي: كيف يمكن تطوير تعليم الموهوبين في مصر في ضوء الخبرة اليابانية؟ 


$$
\text { ويتفرع من هذا السؤال أسئلة فرعية، هى: }
$$

ا - ما هية فلسفة وأهداف تعليم الموهوبين في اليابان؟

r- ما أهم طرق اكتشاف الموهوبين في اليابان؟

ץ- ما هو نظام تعليم الموهوبين في اليابان؟

ع- ما الرؤية المستقبلية لتطوير تعليم الموهوبين في مصر في ضوبي ضوي الخبرة

اليابانية؟

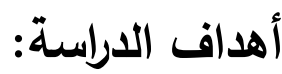

تهدف الدراسة الحالية إلى:

1 - ما هية فلسفة وأهداف تعليم الموهوبين في اليابان؟

r- ما أهم طرق اكتشاف الموهوبين في اليابان؟

ץ-وضع تصور لرؤية مستقبلية لتعليم الموهوبين في مصر في ضوه الخبرة

اليابانية؟ و

أهمية الدراسة: تكمن أهمية الدراسة الحالية في النقاط التالية:

ا-ضرورة مواكبة الخبرات العالمية المعاصرة، التي تهتم بتعليم الموهوبين

ورعايتهم.

r-قد تفيد الدراسة المسئولين عن تطوير التعليم في مصر عند إعداد، وتطوير

سياسة التعليم الخاصة بالموهوبين.

ץ-توجيه الباحثين، والدارسين، وواضعي المناهج بواقع الموهوبين ، والمحاولة

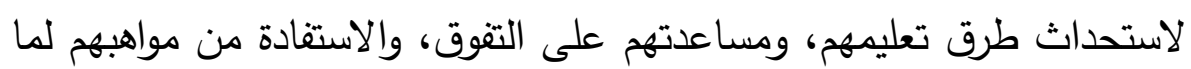

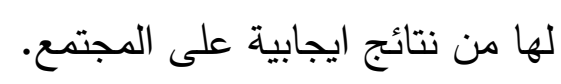

منهج الدراسة:

استخدمت الباحثة المنهج الوصفي

$$
\text { إجراءات الدراسة: }
$$

تتم إجراءات الدراسة من خلال الاجابة على تساؤلات الدراسة كالتالي: 
التساؤل الأول: " ما هية فلسفة وأهداف تعليم الموهوبين في اليابان؟"

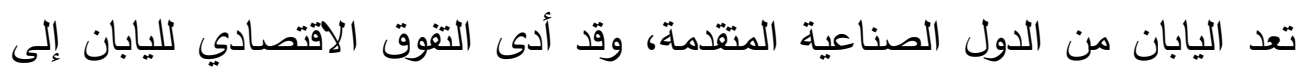
جذب الكثير من الانتباه نحو نظامها التعليمي، واليابان تعتمد في نظامها التعليمي على

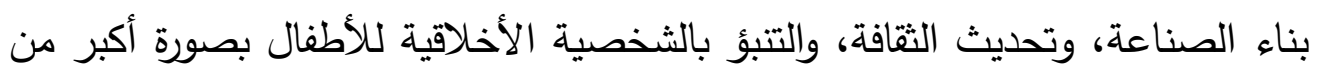
أي دولة أخرى(Thomas, 1993,404) . كما تعتبر اليابان من أول دول العالم في إنتاج السفن، وأجهزة المذياع، والثانية في إنتاج السيارات ومنتجات المطاط، والثالثة في

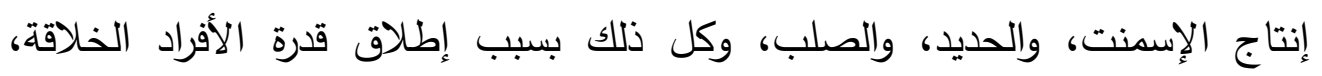

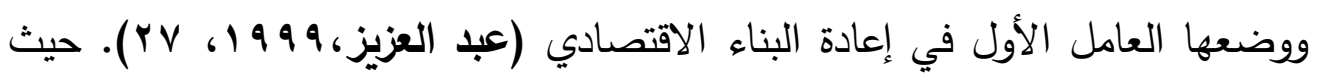
تعتمد فلسفة رعاية الموهوبين في اليابان على النقاط التالية:( محمد وعبد

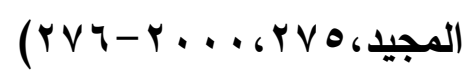

1-عدم وضع قيود على الامتياز ، والتقوق فلا يوجه اللوم، أو النقد إلى فرد يعمل بجد

$$
\text { • تام }
$$

ץ-المساعدة على تتمية مواهب، وقدرات الطفل قبل سنوات الالتحاق بالمدرسة حيث تثار دوافعه، ويشجع بحماس لإظهار مهاراته الابتكارية.

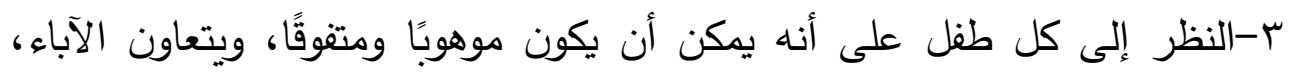
والمدرسة، والمعلمون لتحقيق ذلك ،كما تستخدم طرق تدريس خاصة تتمى التفوق

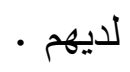

ع-الوعي في تصميم الأدوات التي سيتبعها التلاميذ تحت إشراف الآباء لتنىى

$$
\text { الابتكارية والإبداع، وإظهار مواهبهم . }
$$

ه-يهتم المعلمون في اليابان بالموهوبين، والمتفوقين عن طريق تتمية الفنيات، والمهارات عند جميع الطلاب، وكذلك الابتكارية . 4-التدريب الجماعي حيث يتم الاهتمام بتدريب الفريق على الابتكارية . V-تعاون الآباء، والمعلمين فى تتمية المهارات يؤدى إلى الابتكارية. 1-البحث الدائب عن أفكار ، وإبداعات جديدة. 
9-التعلم بالتوجيه الذاتي، وينتشر هذا النوع من التعليم في اليابان وله تقديره أيضًا. • ا-النظرة البعيدة التي تطبع المستقبل للثقافة اليابانية بطابع يؤثر بدرجة كبيرة على لـ تعليم العباقرة والمتفوقين من المجتمع الياباني. وهنا يمكن الإثارة إلى وجود مدارس خاصة باليابان تعتنى بتربية الذكاء، والهدف منها

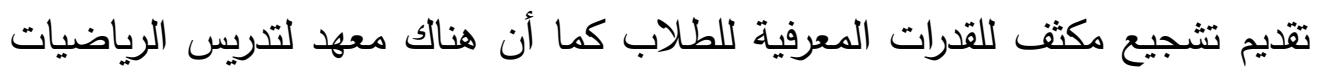

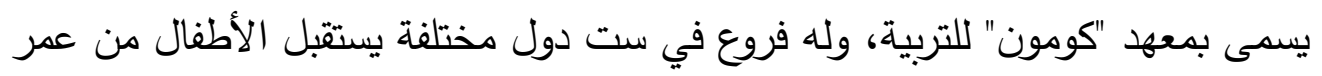

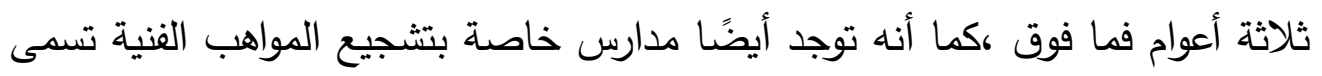

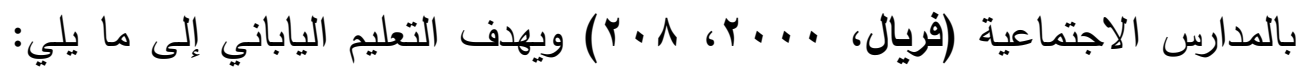
(http: // www. Sagifted.com)

1- الاهتمام المبكر بتتمية قدرات التفكير الأساسية منذ الطفولة المبكرة . r- الابتعاد في التعليم عن حشو الذهن بالمعارف لأن ذلك يعوق التفتح الحقيقي لقدرات التفكير الإبداعية الأساسية. r-تهذيب وتتمية قدرات واسعة ومتتوعة لدى الفرد وعدم الاقتصار على بعض

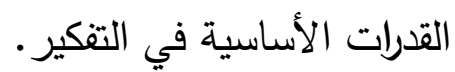
ع - الاهتمام بتنمية القدرات الأساسية في التفكير التي يحتاجها الطفل في حياته المستقبلية بصورة خاصة. وتؤكد هذه الأهداف على الاهتمام بالقدرات الأساسية لدى الطلاب، وتتميتها وأيضًا الاهتمام بتنوع القدرات الإبداعية عند الطلاب كما تؤكد أيضًا على الابتعاد عن حشو الذهن بالمعارف لإتاحة الفرصة لظهور المواهب، وبالتالي تتميتها. حيث يعد الهدف من تعليم الموهوبين، والمتفوقين في اليابان هو مساعدة هؤلاء الطلاب على تنمية مهاراتهم الخاصة سواء كان ذلك في المجالات الأكاديمية، أم الفنون المختلفة، أم هُعاء المجالات الأخرى للنمو، والتعلم، ويتم تقديم التدريب الخاص لهح بواسطة المعلمين، والمدربين سواء فى المنزل، أم في المؤسسات الخاصة. (Takeuchi,1997,6)

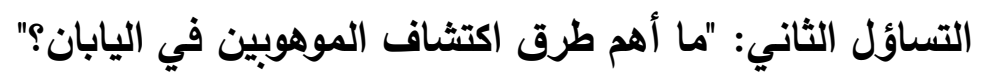


يعتمد نظام التعليم الياباني على الترفيع الآلي حتى نهاية المرحلة المتوسطة "الإعدادية" ثم تعقد مسابقات في نهاية هذه المرحلة لتحديد مسار الطالب، ونوع التعليم الذي الذي لإي سيلتحق به الطالب. وتعتبر الاختبارات التحصيلية المحك الأساسي للانتقال إلى في المراحل الدراسية العليا، وكنلك في تحديد نوع المهن التي سيلتحقون بها طول حياتهم، وكانت هذه الاختبارات تعقد في صورة امتحانات عامة كل عام فى جميع المراحل، وذلك حرصًا على المستوى الأكاديمي المرتفع للطلاب لارجة أن هذه الاختبارات تحدد نوع الجامعة التي يلتحق بها الطالب، وهى مرتبة هرميًا بحيث نجد جامعتي "طوكيو وكيوتى" في القمة يليهما جامعة "توهوكو" حتى نصل إلى قاعدة الهرم التي تضم مجموعة من المعاهد التي تمنح خريجيها دبلومات تخصصية مختلفة ،وبهذا يتنوع التعليم في اليابان ويتشعب بحيث يتناسب مع قدرات الطلاب ومواهبهم. (عبد العزيز، مرجع سابق، I IVV) وتتم آليات اكتثاف الطلاب الموهوبين في اليابان، وتتمية قدراتهم

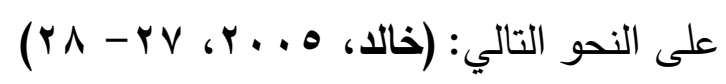

1- البحث عن الطلاب الذين يزيد ذكائهم عن • با ا درجة بمقياس القدرات العقلية.

$$
\text { r- عمل مباريات في التفكير الابداعي لحل المشكلات. }
$$

r- الاهتمام بالاختراعات، وعمل معرض سنوي يسمى نظرة في مستقبل عالم الغد. ع- عقد امتحان قبول للطلاب المبدعين في مختلف المراحل التعليمية، وهذه الاختبارات تشمل( الذكاء، التحصيل، المعلومات العامة، الفحص الطبي،

المقابلة الشخصية).

0- تحديد مدارس خاصة لصفوة الطلاب وتحديد جامعات خاصة للمتميزين من الطلاب الجامعيين، ومن هذه المدارس والجامعات يتم إثارة المنافسة باستمرار بين الطلاب ليحققوا أفضل النتائج لأنهم أمل المجتمع الياباني. 7- إنشاء معاهد خاصة للابتكار والابداع.

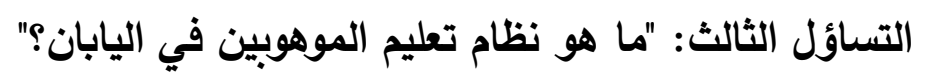


يتخذ نظام تعليم الطلاب الموهوبين في التعليم الثانوي الياباني مجموعة من الأشكال يمكن توضيحها فيما يلي:

\section{1- تنظيم الفصول تبعًا لإنجازات الطلاب :}

تقوم وزارة التربية في اليابان بتشجيع المعلمين على تتظيم الفصول تبعًا لإنجازات

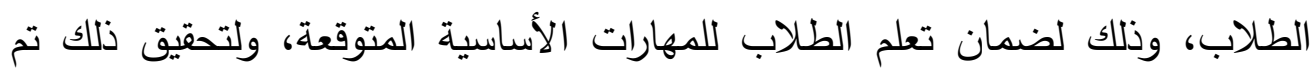
السماح للمدارس المحلية بتطوير ما يسمى بنظام اقتفاء الأثر، والتي يتم فيها وضع

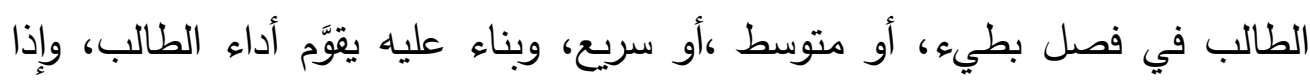
تطور مستوى الطالب البطيء فإنه ينتقل إلى مستوى أعلى،والمحتوى الأساسي للمنهج هو نفسه فى كل المسارات بينما تتنوع السرعة التي يتم بها، ولكي يتم تقديم مساعدة

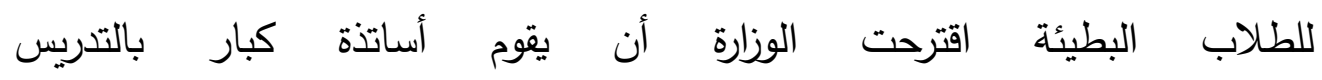
لهم.(Stevenson, 1997,14)

$$
\text { r - التعليم الفردي: }
$$

يقوم هذا النظام على تشجيع المدارس الثانوية لجعل المنهج أكثر مرونة حتى يمكن

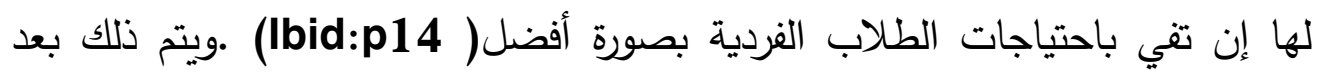
المدرسة، أو ما يطلق عليه Juku أو المدارس الخاصة طول الوقت، أو المدارس الخاصة التي يقضى فيها خريجو المدارس العليا عاما كاملا للإعداد لاختبارات القبول، ونظرًا لأن النجاح في هذه الاختبارات يعتمد على التحصيل، والاجتهاد أكثر من الموهبة فإنه مفتوح أمام جميع الطلاب كما أن هنالك تنافس شديد من أجل الحصول على فرص في مدارس الإعداد أو المدارس الخاصة، وبعد التحاقهم بهذه المدارس يتم

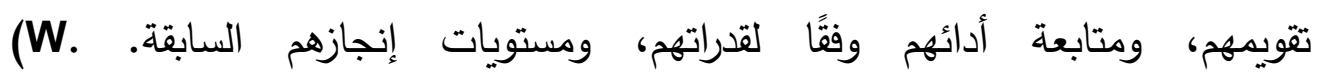

Thomas,404)

r- أنثطة ما بعد المدرسة : 
تتواجد الفرص في المدارس الحكومية للطلاب من أجل إثراء تعليمهم من خلال النوادي، وفصول ما بعد المدرسة، وهذه الأنشطة المنهجية الإضافية مفتوحة لكل الطلاب، حيث يظل طلاب المرحلة الثانوية بعد الدراسة اليومية ساعات عديدة، ويعتمد حجم الأنشطة على حجم المدرسة ويتم فيها تقديم موضوعات متعددة مثل برمجة

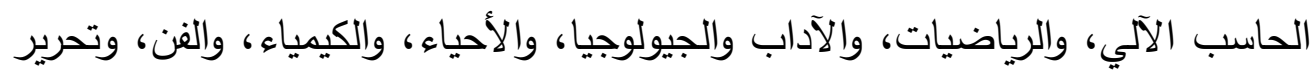
الكتابة في المجلات. ع - البرامج التسريعية:

تعتمد رعاية المتفوقين، والموهوبين من الناحية الأكاديمية في اليابان على سياسة التسريع; فالطلاب الموهوبين يستطيعوا إنجاز المرحلة الثانوية بصفوفها الثلاثة في عامين دراسيين فقط، وبإمكان الطلاب أن يتخطوا الصف الثالث الثانوي بعد أن يجتازوا اختبار للتعرف على مستوى القدرات الأكاديمية، والإبداعية، وبخاصة مادة الفيزياء، ولقد طبق ذلك في عام (999 1) وتم إلحاق ثلاثة طلاب بقسم الفيزياء في كلية التكنولوجيا جامعة شيبا باليابان. (Bahari,2010 ) حيث تعد برامج التسريع من أهم البرامج التي تلقى قبولاً لدى المجتمع الياباني، في رعاية الطلاب المتفوقين الموهوبين ومن أهم الأساليب المطبقة فيها، أسلوب الالتحاق المبكر بالجامعة، وعلى لهي

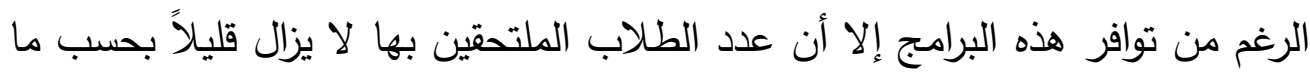

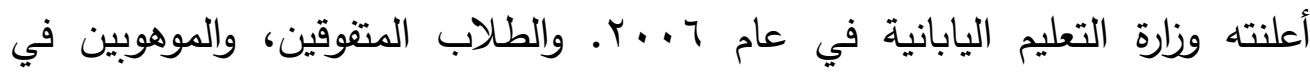
المجتمع الياباني عادة ما يلتحقون بمدارس تعرف باسم Cram School ويدرس الطلاب في هذه المدارس عدد من الكورسات المتقدمة في المجالات المختلفة، الأمر الذي يسهم في دعم عملية التعليم الخاصة بالمتفوقين الموهوبين في خارج أنظمة التعليم

(Shibata,2009,190).العامة

ومن خلال ما سبق تسفر الخبرة اليابانية في مجال رعاية الموهوبين ما يلي: 1- تهتم اليابان بتقديم تعليم مناسب بحيث يكون كل مواطن موهوب ومتفوق.

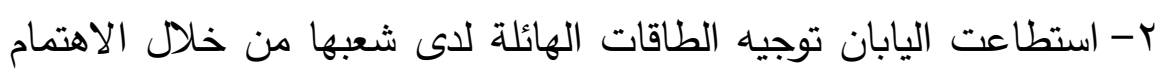

بالموهوبين والمتقوقين. 
r- الاهتمام بتقديم الأنشطة اللاصفيه للموهوبين، والتي تتميز بأنها إجباري

$$
\text { من الصف الرابع الابتدائي. }
$$

צ - تخصيص مدارس، ومعاهد ونوادي للمبتكرين والمبدعين لتتمية قدراتهم،

$$
\text { وعقد مباريات في التفكير الإبداعي لحل المشكلات. }
$$

$$
\text { 0- الأهتمام بإظهار المواهب قبل مرحلة الدراسة. }
$$

1- الأهتمام بالجانب النفسى من خلام عدم تقديم النقد أو اللوم أو العتاب.

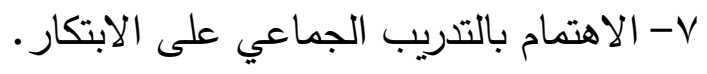

التساؤل الرابع:" ما الرؤية المستقبلية لتطوير تعليم الموهوبين في مصر في ضوء

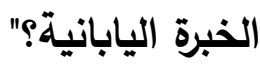
إن الاهتمام بتعليم الموهوبين في مصر يزداد لاعتباره أمرًا حيويًا، وللحصول على نتائج إيجابية لمخرجات تعليم الموهوبين في مصر يتطلب وضع رؤية مستقبلية لتطويره من خلال المدخلات: والمتضمنة بالمدخلات "الفكرية البشرية والمادية والتقنية والمالية"، و العمليات :والمتضمنة بعمليات" الإدارة والتسيير واستراتيجيات التدريس الأنشطة التربوية

$$
\text { أ-الوتهدف من الرؤيلة ما يلي: }
$$

"تعليم الموهوبين باستخدام استراتيجيات متعددة لتعليم الموهوبين في مصر بما يتناسب مع الاتجاهات العالمية المعاصرة في ضوء الخبرة اليابانية."

$$
\text { ب- محاور الرؤية: }
$$

ترتكز محاور الرؤية من حيث الاهتمام بالموهبة على ثلاث محاور أساسية هى: 1-الطالب: التأكيد على تربية كل طالب كموهوب حيث أصبحت نظرة الاتجاه المعاصر أن كل فرد يمتلك قدرات وامكانات خاصة،، وفي نفس الوقت التأكيد على الاهتمام بالموهوبين حتى وإن كان تعليم الموهوبين يشمل الجميع، فإن فئة متميزة يجب الاهتمام بها. بالإضافة إلى الاهتمام بالاكتشاف المبكر للموهبة حتي يمكن تصميم برامج تتاسب احتياجاتهم وتتمي قدراتهم. 
r- المعلم: إن إعداد معلم الموهوبين من أهم عوامل نجاح منظومة تعليم الموهوبين للحصول على مخرجات تعليمية بمقاييس عالمية، ويتم ذلك عن طريق: - تقديم برامج تدريبية شاملة لتطوير القدرات المهنية للمعلم - أن يكون من الحاصلين على درجة الماجستير والدكتوراه. - تكوين فريق متابعة لتقييم البرامج التدريبية التي تقدم للمعلم. r- الاستراتيجيات التعليمية وإلمناهج:

إن تعدد أنواع المواهب يحتاج إلى تتوع في الاستراتيجيات التي تستخدمها الدوله في تعليم الموهوبين فيصبح لكل موهبه موقف تعليمي خاص بها وذلك يتطلب أن: - - يتم تعليم الطلاب الموهوبين في مصر باستراتيجيات متتوعة كالاسراع، والتجميع ، والاثراء.

- تصميم نظام تعليم قائم على مشروعات يقدمها الطالب بنفسه. - وضع مناهج مخططه لتحفيز عمليات التفكير الابداعي وتبعد عن الحفظ والتلقين.

- - ربط المنهج بالبيئة للتعلم عن طريق المواقف الحياتية الحقيقية.

\section{ج- آليات تنفيذ الرؤيةة:}

1- تعديل اللوائح والتشريعات المنظمه لتعليم الموهوبين: ويتم ذلك عن طريق: - - مراجعة كل القوانين المتعلقة بالموهوبين. - عمل دراسات استطلاعية والاستعانه بخبراء في مجال الموهبة لتطوير اللوائح بما يواكب الاتجاهات العالمية. - وضع تشريعات تحدد مهام ومسئوليات الدولة ومؤسساتها. r- التنمية المهنية لادارة المدرسة والمعلم: ويتم ذلك عن طريق: - - إجراء دراسات لتقويم الاحتياجات التدريبية الفعاله في ظل التغيرات العاليمة. 
- - تحديث الحصر الاحتياجات التدربيية للعاملين في إدارة المدارس والمعلمين

$$
\text { بمدارس الموهوبين. }
$$

- إخخال آليات متتوعة للتمية المهنية مثل التدريب عبر الانترنت، والاستعانه

$$
\text { بخبرات أجنبية. }
$$

- - رصد المبالغ المالية اللازمة لعملية التدريب.

r- تقديم تعليم مناسب لقدرات الموهوبين: ويتم ذلك عن طريق:

الاستعانه بالخبرات العالمية التي نجحت في مجال الموهبه مثل الخبرة اليابانية، ودراسة المناهج والأنشطة والبرامج التي تستخدمها الأنظمه العالمية في تعليم الموهوبين، والتي تتاسب القدرات العقلية لديهم، وتقييم هذه البرامج في ضوء الظروف المحلية بمعايير عالمية، مما يتطلب اتخاذ القرارات بتبني أساليب واستراتيجيات حديثة لتعليم الموهوبين في مصر . ع- بناء الثراكة بين مؤسسات التعليم المختلفة لاعم برامج الموهوبين: ويتم ذلك

$$
\text { عن طريق: - n }
$$

$$
\text { - حصر الجامعات والمعاهد التي تدعم تنفيذ برامج الموهوبين. }
$$

- دعوة مؤسسات التعليم العالي (الحكومي - الخاص) للمشاركة في الأنشطة

$$
\text { والرامج والمسابقات. }
$$

- - عرض المشاريع البحثية في المؤتمرات العلمية المحلية والعالمية.

ه- كسب دعم القطاعات الخاصة في تمويل المشاريع البحثية في مجال الموهبة :

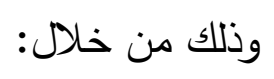

- - عقد لقاءات توعية لرجال الأعمال وأصحاب الشركات والمصانع بشأن أهمية

$$
\text { المشاريع البحثية الخاصة بالموهوبين. }
$$

- - تنظيم أنشطة داخل المدارس لتقوية العلاقة بين القطاع الخاص والحكومي. 
في ضوء دراسة الخبرة اليابانية في مجال الموهبة أسفرت نتائج الدراسة على ما يلي: 1- إصدار القوانين والتشريعات ، ورصد الميزانيات الكبيرة ، لها الدور الأكبر ، والأساسي في حفظ حقوق الطلاب الموهوبين وتقديم أفضل رعاية، وأنسب الطرق التعليمية. r -الاهتمام باكتشاف، ورعاية الطلاب الموهوبين منذ المراحل التعليمية الأولى لمساعدة الموهوبين في تحديد هوية موهبته وصقلها. r- تطبيق أساليب ، واستراتيجيات تعليم متنوعة لرعاية الموهوبين. ع - دعم القطاع الخاص مثل رجال الأعمال، والشركات، وأصحاب المصانع في دعم المدارس التي ترعى الموهوبين، والمتميزين بما يتوافق مع متطلبات، واحتياجات المجتمع، والتي بالتالي تعود بالنفع عليهم، ويكون ذلك في صورة تقديم المنح الدراسية بالجامعات وتمويل مشاريعهم البحثية. 0- الاهتمام بتطوير مناهج الموهوبين لإبراز عمليات التفكير العليا لديهم، والمفاهيم ذات المستوى الراقي لديهم.

4- تطبيق آليات التسريع في تعليم الموهوبين كآلية الالتحاق المبكر بالجامعة. V- النظر لكل فرد متعلم في المجتمع باعتباره صاحب قدرات متتوعة، ومواهب متعددة، ينبغي تتميتها إلى أقصى حدود ممكنه.

حسين كامل بهاء الدين (99V (1). التعليم والمستقبل، دار المعارف، القاهرة. 
خالد خليل الشخيلي( ه . . ب).الأطفال الموهوبون والمتفوقون وأساليب اكتشافهم وطرائق رعايتهم، دار الكتاب الجامعي، الإمارات العربية المتحدة ، العين.

زكريا الثربيني، يسريه صادق (Y . . r). أطفال عند القة..... الموهبة التفوق العقلي الابداع، دار الفكر العربي، القاهرة، كرب،

فريال القحف( . . ب).نماذج من تجارب الدول العربية والأجنبية في مجال رعاية المتفوقين.المؤتمر العلمي العربي الثاني لرعاية الموهوبين والمتفوقين "التربية الإبداعية أفضل استثمار للمستقبل "المجلس العربي للموهوبين والمتفوقين،الأردن:عمان. عبد العزيز السيد الثخص (•99 ().الطلبة الموهوبون في التعليم العام بدول الخليج العربي،أساليب اكتشافهم وسبل رعايته ،الرياض، مكتب التربية العربي لدول الخليج.

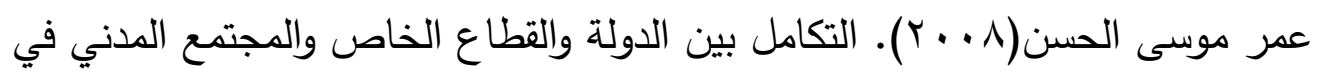
تربية الموهوبين ورعايتهم، ورقة عمل مقدمه للمؤتمر السادس لوزراء التربية والتعليم

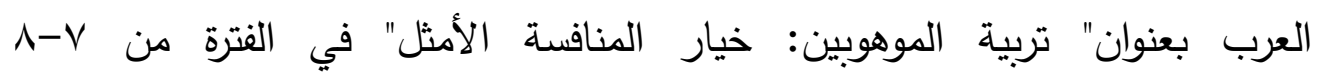

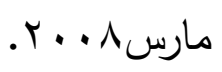

محمد بن عبد المحسن التويجري،عبد المجيد سيد أحمد منصور ( . . ب):الموهوبون آفاق الرعاية والتأهيلبين الواقعين العربي والعالمي.الرياض: مكتبة العبيكان. محمد رمضان شعيب(· ( • ( ). مناهج تربية الموهوبين والمتفوقين"المنهج الاثرائي

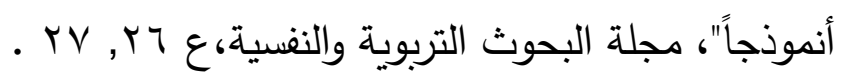
ثانيًا: المراجع الأجنبية

Bahari, mohd fathil(2010), A Reflection: The Japanese Approach to Gifted and Talented Students. http: \\ fathil blogspot.com \ 2010\12 reflection- Japanese- Approach to gifted. Html. 
Shibata, \& Forbes, Dianna(2009). teachers and counselors perspectives on gifted children and gifted education: New Zealand and Japan. Gifted Education International, Vol 25.

Stevenson Harold W.,et al(1997) :Education of Gifted and Talented Students in China, Taaiwan, and Japan ,Office of Educational Research and Improvement (ED), Washington, DC, Jun .

Takeuchi, Michio(1997) :Several Controversial Issues on Early Childhood Education and Care in Contemporary Japan ,US .Department of Education.

Thomas, Southern W., et al.(1993). "Acceleration and Enrichment : the Context and Development of Program Options", In : Kurt A. Heller, et al. (Eds.), International Handbook of Research and Development of Giftedness and Talent, (New York: Pergamon Press Ltd).

ثالثًا: المواقع الالكترونية

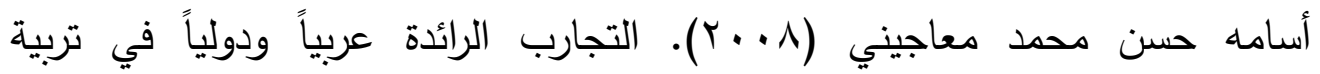
الموهوبين ورعايتهم-،دراسة نظرية مسحية- مقدمة للمؤتمر السادس لوزراء التربية

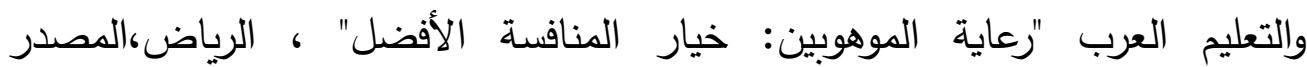
r.http:old.edu.salpagelarl39041 http: // www. Sagifted.com/ Pb/idex.php (2-7-2017 